\title{
The improvement of uncertainty measurements accuracy in sensor networks based on fuzzy dempster-shafer theory
}

\author{
Ehsan Azimirad a,1, , Seyyed Reza Movahhed Ghodsinya ${ }^{\mathrm{a}, 2}$ \\ a Electrical Engineering Department, University of Torbat Heydarieh, Torbat Heydarieh, Iran \\ ${ }^{1}$ e.azimi@torbath.ac.ir; ${ }^{2}$ sr.movahhed@torbath.ac.ir \\ * corresponding author
}

\section{ARTICLE INFO}

\section{Article history}

Received January 27, 2020

Revised May 21, 2020

Accepted June 1, 2020

Available online July 12,2020

\section{Keywords}

Sensor Networks

Fuzzy Dempster-Shafer Theory

Model-Based Uncertainty

Fuzzy Sets Theory

Combat Management System

\section{ABSTRACT}

Threat Assessment is one of the most important components in combat management systems. However, uncertainty is one of the problems that occur in the input data of these systems that have been provided using several sensors in sensor networks. In literature, there are some theories that state and model uncertainty in the information. One of the new methods is the Fuzzy Dempster-Shafer Theory. In this paper, a modelbased uncertainty is presented in the air defense system based on the Fuzzy Dempster-Shafer Theory to measure uncertainty and its accuracy. This model uses the two concepts naming of the Fuzzy Sets Theory, and the Dempster-Shafer Theory. The input parameters to sensors are fuzzy membership functions, and the basic probability assignment values are earned from the Dempster-Shafer Theory. Therefore, in this paper, the combination of two methods has been used to calculate uncertainty in the air defense system. By using these methods and the output of the Dempster-Shafer theory are calculated and presented the uncertainty diagrams. The advantage of the combination of two theories is the better modeling of uncertainties. This makes that the output of the air defense system is more reliable and accurate. In this method, the air defense system's total uncertainty is measured using the best uncertainty measure based on the Fuzzy Dempster-Shafer Theory. The simulation results show that this new method has increased the accuracy to $97 \%$ that is more computational toward other theories. This matter significantly increases the computational accuracy of the air defense system in targets threat assessment.

This is an open access article under the CC-BY-SA license.

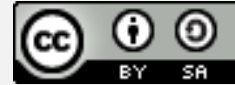

\section{Introduction}

Battle Management System is an instruction control and data system that creates a photo of the operational environment provides decision-making and execution operation in sensor networks [1]. In an operational environment, it needs to protect the assets against the enemy. This work is being done by assigning the weapons systems in the combat management system [2]-[6]. The general job of a battle management system is to data fusion, target recognition and detection, management of weapon, and decision-making. The decision-making is automatically mounted on the battle management system consists of three parts. (1) Sensors as the system input, (2) Command, and Control as the heart of the system and (3) weapons as the system output.

The main task of the command and control system is data integration. In this system, the kind of sensors and their numbers are very various. Data fusion techniques are used to increase the capability of sensors in a combat management system. Data fusion is the software part of that system. The concept 
of Data fusion is the process of getting information from multiple sensors to give a complete description of an operational environment. The combination of information has many applications in defense systems, and linear and nonlinear control of systems, and information systems [3].

In a military setting, it is frequently an instance in which real-time decision-making has to appraise the tactical position and support assets against the enemy by allocating weapon systems to them. The dynamic targets are moving and change in their treatment. The different effects are considered for a decision making completed via human intelligence [4]-[6].

In a position by multiple threats, it is crucial to prioritize the degree of threat and the uncertainty of the system. This position represents the friendly assets, and its threat degree indicates the amount of their risk [7]-[9]. The threat degree, introduced as threat value, can be applied to help for intelligent management of sensors, by assigning more resources of sensors to targets by high threat values. To specify which of multiple threats that demonstrate the most danger is of high significance, since errors such as prioritizing a slower threat as a more top threat can lead to the wrong threat. This matter mostly will have intense subsequences. Threat assessment is a high-level data integration procedure. Threat assessment is related to level 3 of the JDL model in the data fusion system [10]-[15].

There are many applications in the literature to using the Fuzzy Dempster-Shafer Theory. These applications have been expressed in the field of threat assessment, risk assessment, data fusion in sensor networks, health assessment in medicine, and many other applications. Investigating the uncertainty of data sensors to achieve proper accuracy in data fusion and reducing this uncertainty is one of the challenges in data fusion in sensor networks [16]-[20].

In this paper, We presented a new method for improvement of accuracy in uncertainty measurements in imperfect information for an air defense system. In this method, the calculation of uncertainty is done using Fuzzy Dempster-Shafer Theory or Fuzzy Evidence Theory. This method uses from the two concepts naming of the Fuzzy Sets Theory and the Dempster-Shafer Theory that the input parameters to sensors are fuzzy membership functions and the basic probability assignment values are earned from Dempster-Shafer Theory [21]-[30].

The remainder of this paper is established as follows. In part 2, the proposed method consists of the concept of uncertainty, detailed explanation of the Generalized Fuzzy Dempster-Shafer Theory, and the suggested modeling. The results and discussion are presented in part 3. In the end, this paper summarized and conclude the finding in the conclusion part.

\section{Method}

\subsection{The concept of uncertainty}

Sensor networks produce extensive data and information that be used to demonstrate the uncertainty of the target [20]. In this information, there is a unique uncertainty that depends on the nature of goals. Assets are challenging to get the mathematical model by using the sensor's information as input and generating the threat value as output. Three types of uncertainty are defined in the literature [31]:

- Fuzziness: the vague boundaries of fuzzy sets.

- Non-specificity: the cardinalities of various sets.

- Discord: the conflict among the sets.

Fig. 1 shows these three types of uncertainty. Due to the incomplete provided information, different types of defects may be considered, such as imprecision and uncertainty. In recent years, many theories have been improved to deal with this type. One difficulty in these methods is quantifying the assurance levels, and one of the new theories to solve this problem is named Generalized Fuzzy Dempster-Shafer Theory (GFDST). 


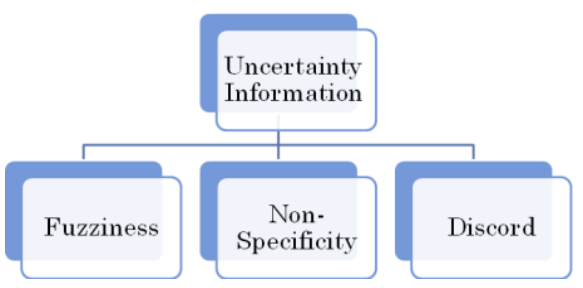

Fig. 1. The three types of uncertainty information

Fig. 2 shows that uncertainty is a subset of threat assessment challenges in the multi-sensor data fusion system. Fig. 2 states that the uncertainty is the imperfection of information sources and ambiguity in human behavior in data fusion systems and sensor networks [31].
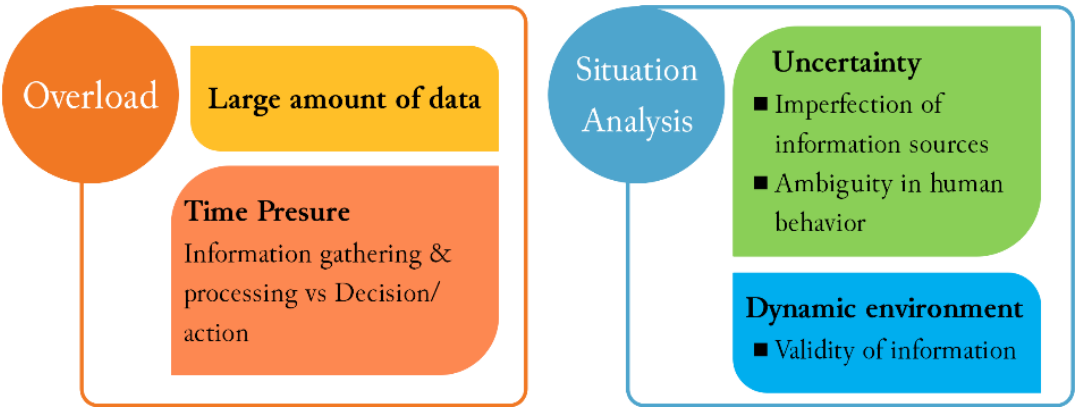

Fig. 2. A subset of challenges of threat assessment naming uncertainty.

There are several theories for the demonstration of uncertainty in sensor networks. These theories are Fuzzy Sets Theory, Evidence Theory, Rough Sets Theory, Probability Theory, and Random Sets Theory. As seen in Fig. 3, each of the theories will be suitable for one particular kind of incomplete data. In really, complicated positions initiate the processing of multiple pieces of information, each describing one or more types of incompleteness.

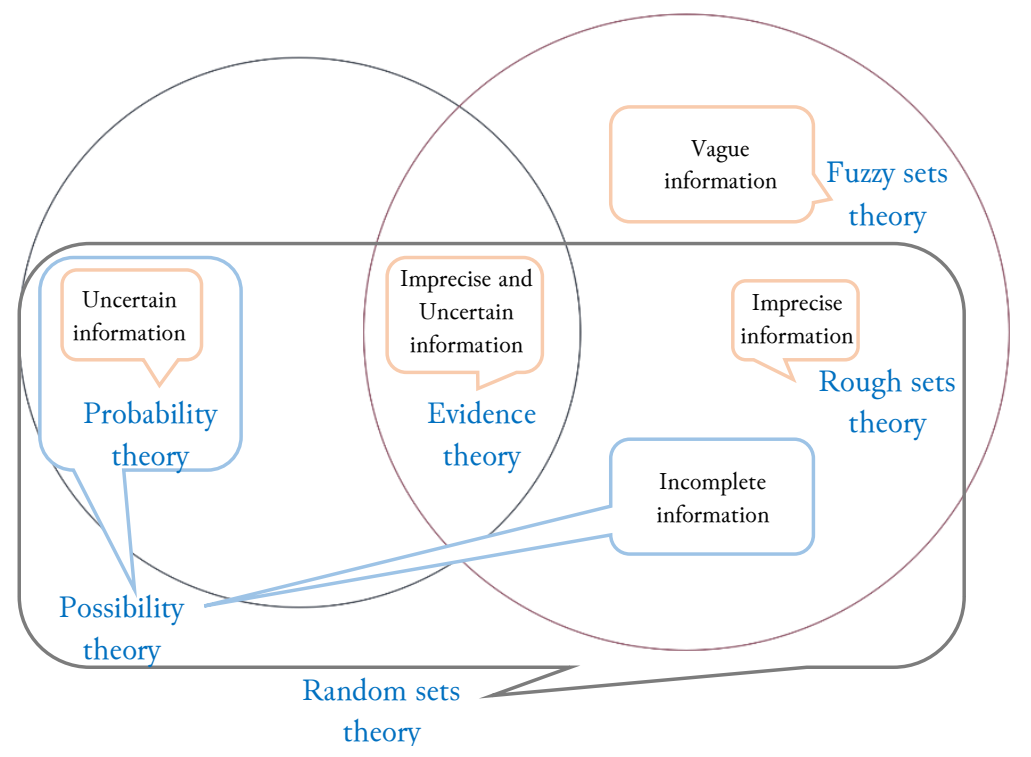

Fig. 3. The types of theories in the modeling of uncertainty.

For example, the fuzzy sets theory is suitable for modeling vague information, or, the evidence theory is ideal for modeling imprecise and uncertain information [32]-[35]. In this paper, a combination of two theory naming Fuzzy Sets Theory and Evidence Theory is used. One of the newest theory is named Fuzzy-Evidence Theory (FET) that is a theoretical body for demonstrating uncertain data that causing all of the three type's uncertainty is obtained. 


\subsection{Generalized Fuzzy Dempster-Shafer Theory}

As it is seen in the introduction part, in twisted military positions; must be presented a particular method for calculation of uncertainty in data fusion systems. This method is gotten as a combination of the mentioned approaches. The integration of various theories is vital, but it has to be done carefully to optimize the lack of information. Therefore, the only method cannot be used with these complicated circumstances. Generalized Fuzzy Dempster-Shafer Theory (GFDST) is a comprehensive theory to show irresponsible information and models all the three types of uncertainties in it. GFDST is based on two basic theories: Dempster-Shafer Theory (DST) and Fuzzy Sets Theory (FST) [35].

Dempster-Shafer Theory uses the concept of evidence, which shows all accessible information about the specific system in terms of a combination of crisp sets and their beliefs. Evidence theory is usually introduced as a theory of generalization of presumption. This theory can present both imprecision and uncertainty [32].

In the DST, the uncertainty is gotten from the function called basic probability assignment (BPA), which is shown in (1). $\mathrm{P}\left(X^{\prime}\right)$ Denotes the power set of $X^{\prime}$.

$$
m: P\left(X^{\prime}\right) \rightarrow[0,1]
$$

A Fuzzy Sets Theory (FST) [23] [25] [33] is a combination of roots in which elements have a degree of membership. The fuzzy number $A=[a, b, c]$ is triangular that be shown in (2).

$$
\mu_{A}\left(x^{\prime}\right)= \begin{cases}\frac{x-a}{b-a}, & a \leq x \leq b \\ \frac{c-x}{c-b}, & b \leq x \leq c\end{cases}
$$

The $\alpha$-cuts for the mentioned fuzzy number in (2), is in below that $\alpha \in[0,1]$.

$$
{ }^{\alpha} A=[a+\alpha(b-a), c-\alpha(c-b)]
$$

In FST theory, one type of uncertainty is represented as a fuzziness [35]. GFDST uses the concepts of the DST and FST to combining all of the uncertainties. Fig. 4 demonstrates this concept.

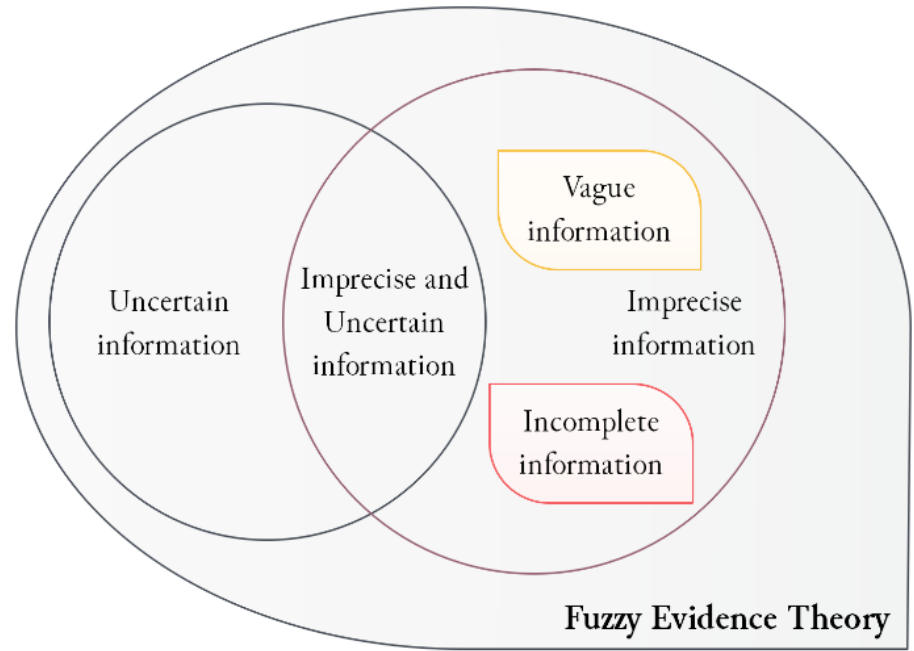

Fig. 4. The concept of fuzzy evidence theory in modeling of uncertainty

\subsection{The measures of uncertainty in GFDST}

A scale of uncertainty is proposed in Generalized Fuzzy Dempster-Shafer Theory called General Uncertainty [35]. General Uncertainty (GM), is defined as follows: 


$$
G M(F B o E)=\sum_{x \in X}\left[\operatorname{Bet} P(x) \log _{2} B e t P(x)+\overline{B e t P}(x) \log _{2} \overline{B e t P}(x)\right]
$$

where

$$
\begin{gathered}
\operatorname{BetP}(x) \equiv-\sum_{i=1}^{f} \frac{m_{X}\left(\tilde{A_{i}}\right) \mu_{\tilde{A}_{i}}(x)}{\sum_{x^{\prime} \in S_{\tilde{A}_{i}}} \mu_{\tilde{A}_{i}}\left(x^{\prime}\right)} \\
\overline{\operatorname{BetP}}(x) \equiv-\sum_{i=1}^{f} \frac{m_{X}\left(\tilde{A_{i}}\right)\left(1-\mu_{\tilde{A}_{i}}(x)\right)}{\sum_{x^{\prime} \in S_{\tilde{A}_{i}}} \mu_{\tilde{A}_{i}}\left(x^{\prime}\right)}
\end{gathered}
$$

$F B o E$ is make of membership function, membership degree, and BPA. Another scale of uncertainty for the Generalized Fuzzy Dempster-Shafer Theory is called Hybrid Entropy. Hybrid Entropy is defined as follows [35]:

$$
F H(F B o E) \equiv-\sum_{i=1}^{f} m_{X}\left(\tilde{A_{i}}\right) \log _{2}\left(m_{X}\left(\tilde{A_{i}}\right)\left(1-F\left(\tilde{A_{i}}\right)\right)\right)
$$

where

$$
F(\tilde{A}) \equiv \frac{1}{\left|S_{\tilde{A}}\right|} \sum_{x \in S_{\tilde{A}}} \frac{\mu_{\tilde{A} \cap \overline{\bar{A}}}(x)}{\mu_{\tilde{A} \cup \bar{A}}(x)}
$$

According to Azimirad and Haddadnia [35], the above measures calculate all types of three uncertainty.

\subsection{The measures of uncertainty in FST and DST}

There are two measures of uncertainty in the fuzzy sets theory that is named non-specificity. The first measure is the model of non-specificity uncertainty in classic sets, the second is the model of nonspecificity uncertainty in the fuzzy sets theory [24][31]. The non-specificity uncertainty in two sets are defined as follows:

$$
\begin{aligned}
& U(A)=\log [1+b-a] \\
& U(B)=\frac{1}{h(B)} \int_{0}^{h(B)} \log _{2}\left[1+B_{2}(\alpha)-B_{1}(\alpha)\right] d \alpha
\end{aligned}
$$

where $A$ is a classic set that is defined in $[a, b]$, and $B$ is a fuzzy set that a,b,c are the coefficient of fuzzy number B. In (10) is used from the concept of height and alpha cut B.

\section{Results and Discussion}

\subsection{Uncertainty Modeling}

In this section, the proposed method for uncertainty modeling based on the fuzzy sets theory and Fuzzy Dempster-Shafer Theory is presented. In one model, model-based uncertainty using fuzzy sets theory and in the second model, model-based uncertainty using fuzzy evidence theory are presented. Fig. 5 and Fig. 6 illustrate the model-based uncertainty using fuzzy sets and fuzzy evidence theories. Firstly, membership functions are converted into fuzzy numbers. The $\alpha$-cut and their original elements are then obtained. Then, the core elements are converted into BPAs, and BPAs are combined based on the Dempster composition rule. Finally, the threat ranking targets are done using TOPSIS algorithm. The output of the model gives threat ranking and uncertainty for air targets. For verifying the proposed method, the uncertainty measures based on FST and FET are calculated [27]-[30]. 


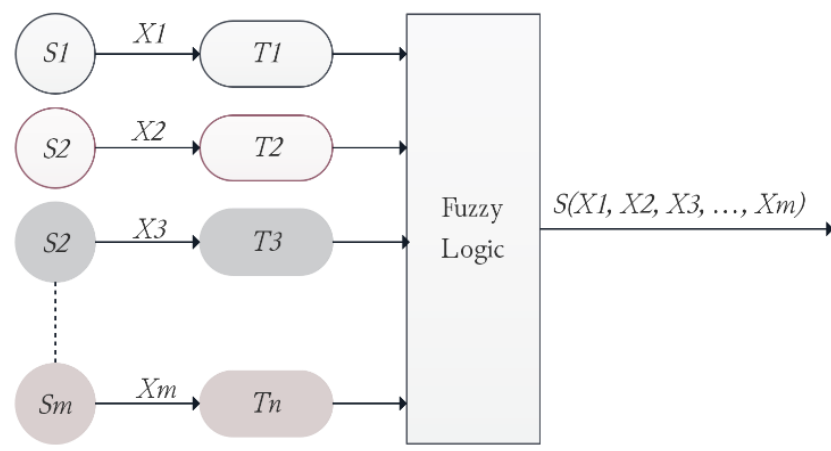

Fig. 5. The model-based uncertainty using fuzzy sets theory

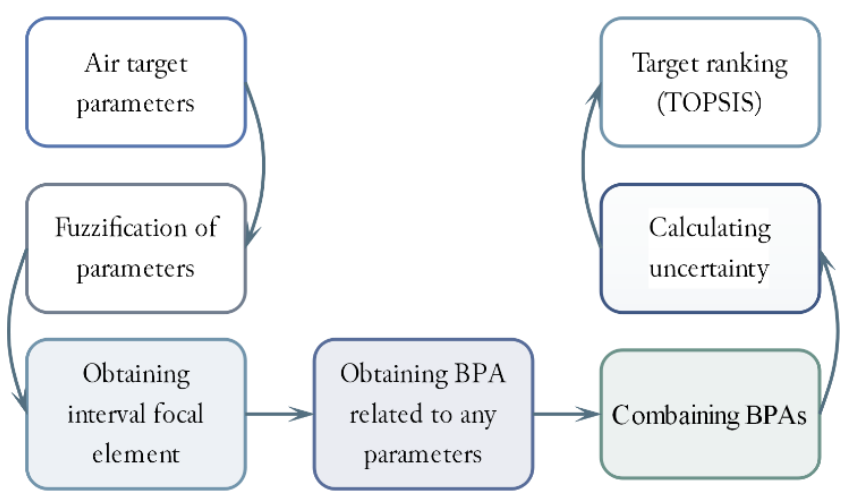

Fig. 6. The uncertainty modeling in target threat assessment.

In Fig. 6, the model-based uncertainty for air defense system based on Generalized Fuzzy DempsterShafer Theory (GFDST) is presented. This model uses two concepts consisting of interval focal elements and basic probability assignment. The first concept is $\alpha$-cuts for the fuzzy number based on (3). The second concept is the basic element for the expression of uncertainty in GFDST based on (1). The proposed method includes four steps that are stated in the following.

\section{Step 1. The Attributes of Target Parameters}

The major work of a battle management system is target recognition and detection. In this system, the decision is spontaneously installed on the air target and others. The diversity of parameters for targets are suggested [35]. The parameters and their descriptions that are used in the simulation are presented in Table 1.

Table 1. The Air Target Parameters.

\begin{tabular}{cc}
\hline Attribute & Description \\
\hline Speed & Proximate speed. \\
Altitude & Proximate foot above the ground. \\
Range & The distance from the target. \\
CPA & Closest Point of Approach that suggested path. \\
Weapon Envelope & The situation with its suggested weapons cover. \\
Own Support & Accessibility of closely friendly. \\
Visibility & A proof of atmospheric status. \\
Maneuvers & Represent the number of maneuvers. \\
Fire & The enemy fire toward asset \\
IFF Mode & Recognize a friend or foe or impartial. \\
Target Support & Accessibility enemy targets. \\
\hline
\end{tabular}


Step 2. Get the Membership Functions

In this section, the system's membership functions based on the fuzzy Dempster-Shafer Theory and BPAs are suggested in relation to input parameters [19][20]. The most important of these parameters are represented in Fig. 7. The other parameters are singleton.

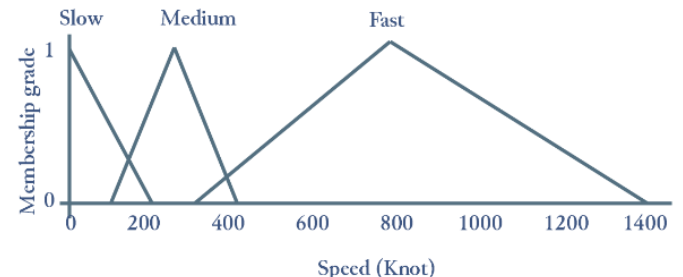

(a)

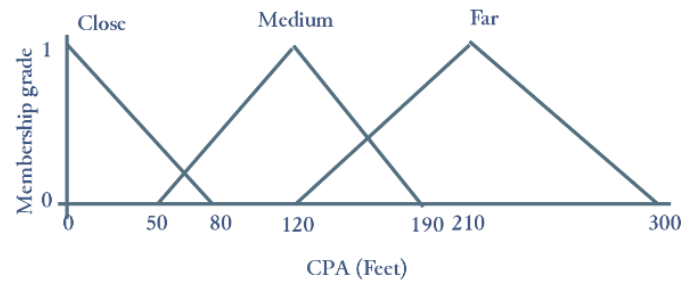

(c)

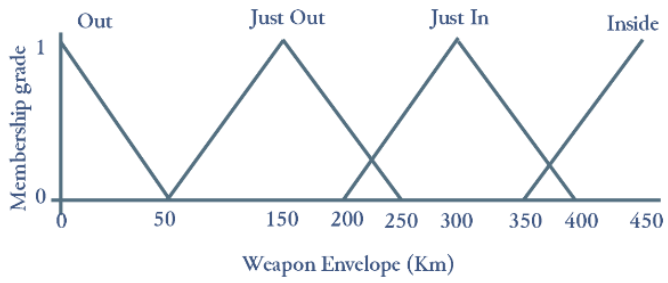

(e)

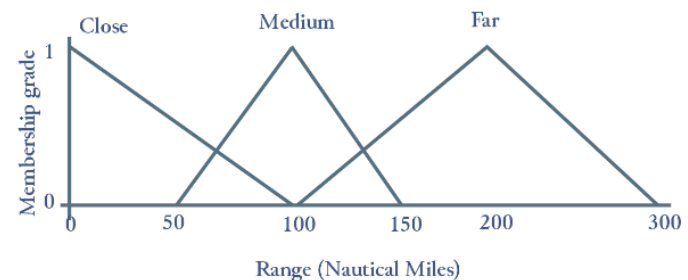

(b)

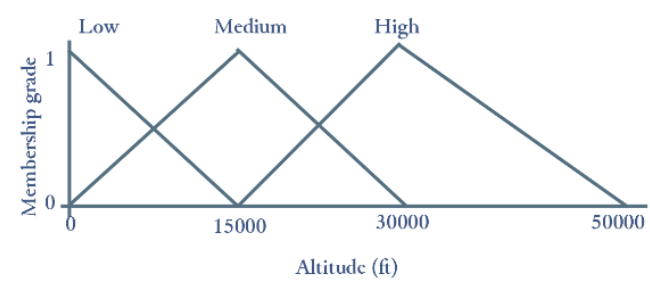

(d)

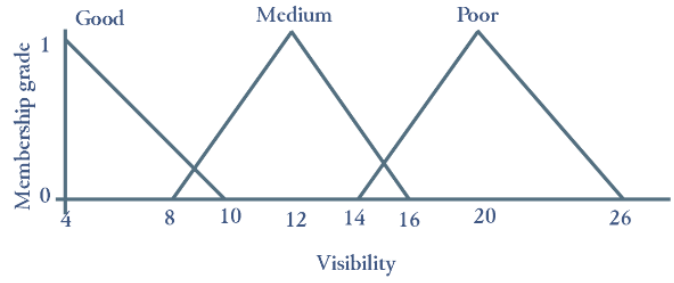

(f)

Fig. 7. Membership functions for triangular input parameters: (a) speed, (b) range, (c) CPA, (d) altitude, (e) weapon envelope, (f) visibility.

Step 3. Get the BPAs

In this section, the fuzzy numbers in (2) and $\alpha$-cuts in (3) are calculated. Then, BPAs in related to the functions in Fig. 7 are obtained. Next, general BPAs are calculated, and the BPAs in related to the functions in Fig. 7 are earned as the following equation [24] [34]:

$$
m\left({ }^{\alpha} A_{i}\right)=\frac{\int_{{ }^{\alpha} A_{i_{\text {lower }}}}^{{ }^{\alpha} A_{i_{\text {inper }}}} \mu_{A}(x) d x}{\sum_{i} \int_{{ }^{\alpha} A_{A_{\text {ilower }}}}^{\alpha_{i_{\text {ipper }}}} \mu_{A}(x) d x}
$$

where ${ }^{\alpha} A_{i}$ lower and ${ }^{\alpha}{ }_{A_{i}}$ upper are bounds in (3).

\section{Step 4. Get of Uncertainty}

Evidence and fuzzy set theories that make fuzzy evidence theory are proper for modeling of uncertain and imprecise information. Therefore, FET can be more useful for modeling of imperfect information and calculation of uncertainty. To calculate total uncertainty in the air defense system based on fuzzy sets theory and fuzzy evidence theory in Fig. 5 and Fig. 6, the uncertainty measures in two theories are used. These measures are in (9) and (10) [20] [31]-[35].

\subsection{Definition of Scenarios}

The four scenarios in this paper for start simulation are defined in Fig. 8 that related to the air defense system in the combat environment. The first scenario states that air target 1 is getting closer to asset 1 . It means the threat is increasing. The threat is decreasing stated in the second scenario states when 
target 2 gets away from the assets. The third scenario states that air target 3 gets away from asset three and then getting closer to its, which describes the threat initially is decreasing and then increasing. The fourth scenario states that air target three initially getting closer to asset one and then get away from it which shows the threat initially is increasing and then decreasing.

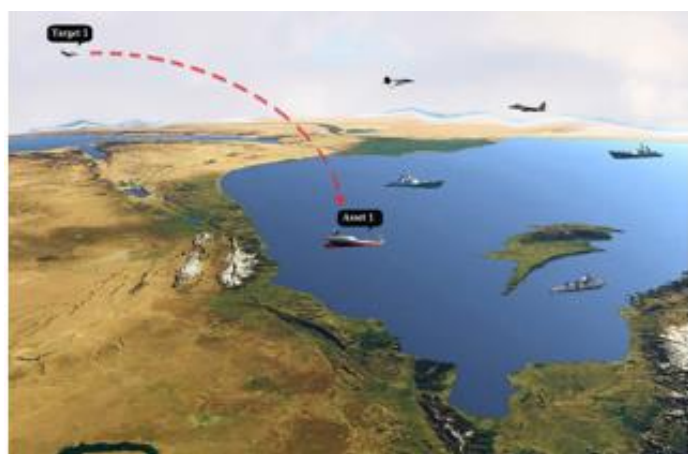

(a)

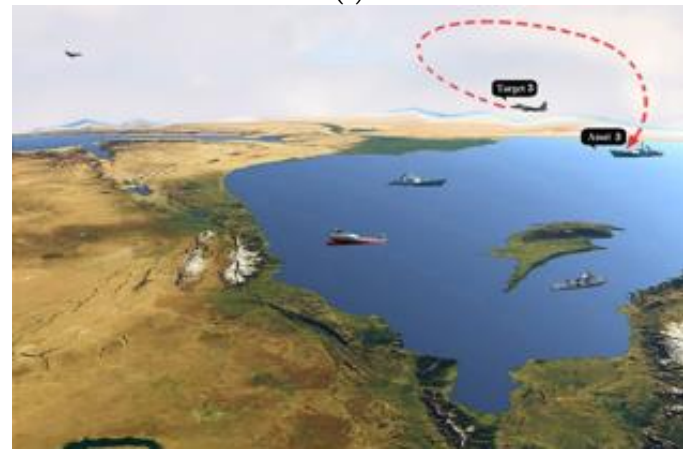

(c)

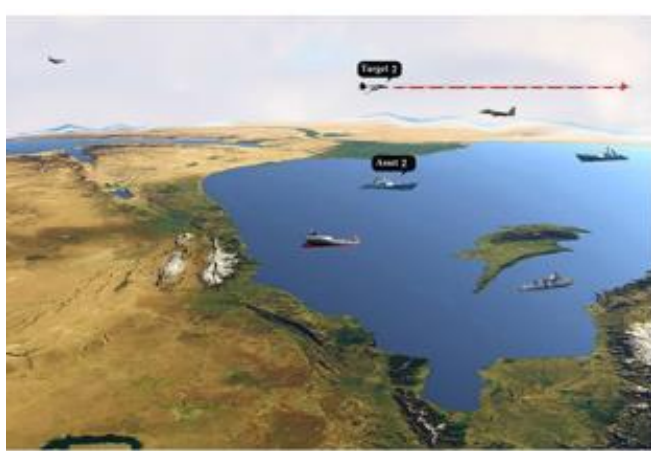

(b)

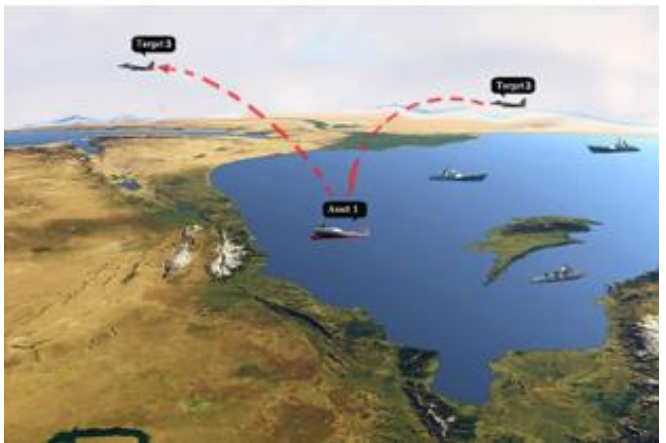

(d)

Fig. 8. The four scenarios in the battle: (a) First Scenario, (b) second Scenario, (c) third Scenario and (d) Fourth Scenario

\subsection{Simulation Results}

Evidence and fuzzy set theories that make Generalized Fuzzy Dempster-Shafer Theory (GFDST) are appropriate for modeling of uncertain and imprecise information. Therefore, GFDST can be more useful for modeling of imperfect information and calculation of uncertainty. To calculate total uncertainty in the air defense system that is based on fuzzy sets theory and GFDST in Fig. 5 and Fig. 6, the uncertainty measures in FST and GFDST theories are used. These measures are in (4), (9), and (10). In this stage, the simulation results for the calculation of uncertainty in the air defense system in the below combat scenarios using fuzzy sets theory and GFDST are stated. Fig. 9 shows the result of applying uncertainty measures using FST. In this figure, the real-time variations of the two uncertainties are demonstrated.

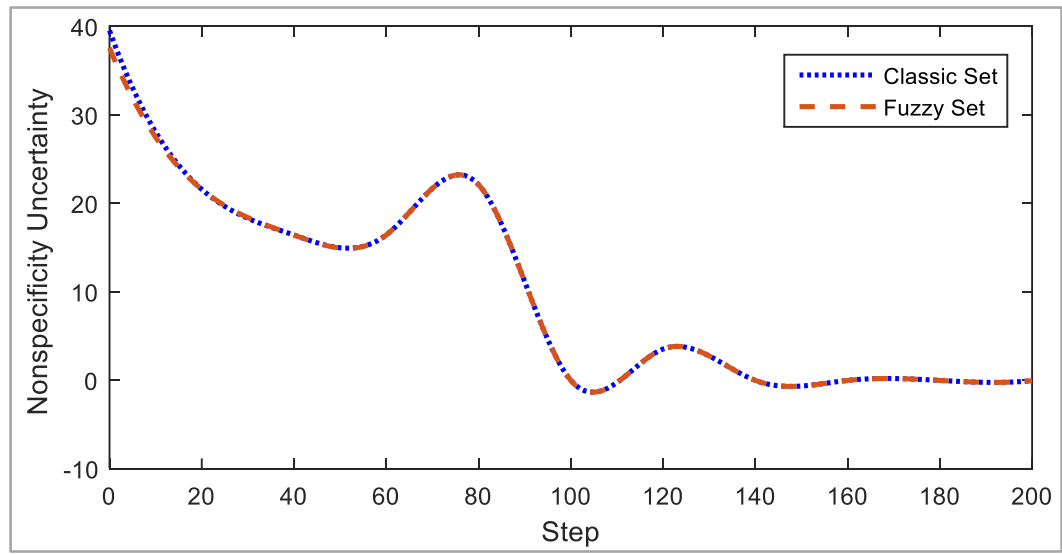

Fig. 9. The total uncertainty based on Fuzzy Sets Theory 
Fig. 10 shows the result of applying uncertainty measures using GFDST. In this figure, the real-time variations of general uncertainty is demonstrated.

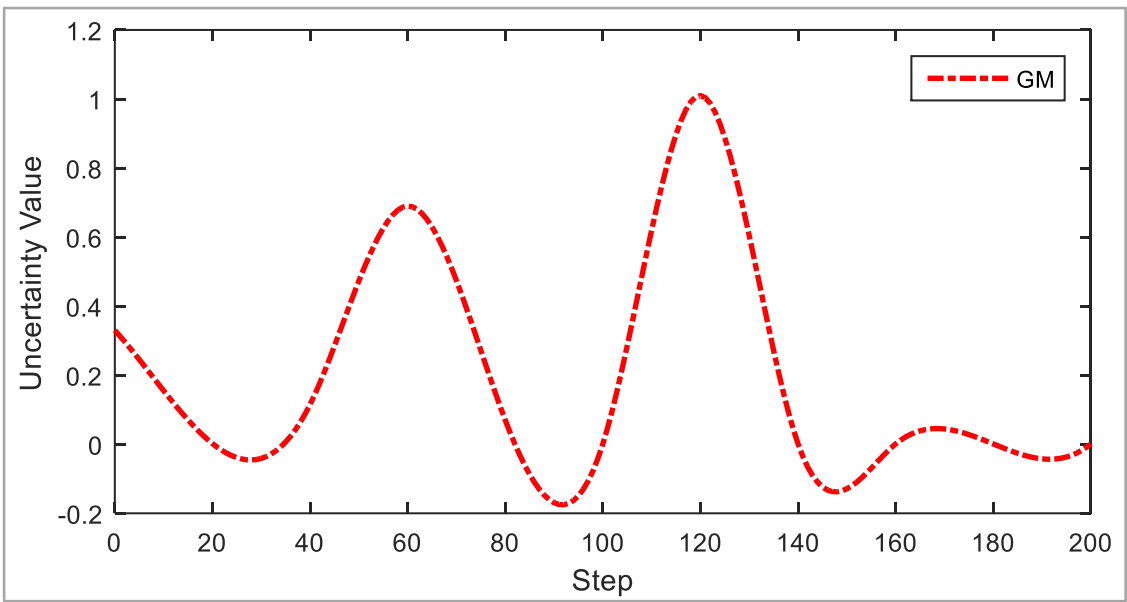

Fig. 10. The total uncertainty based on Generalized Fuzzy Dempster-Shafer Theory

Fig. 9 and Fig. 10 state that the general uncertainty in GFDST is much better than non-specificity uncertainty in FST and classic sets. Fig. 11 and Table 2 demonstrate this matter.

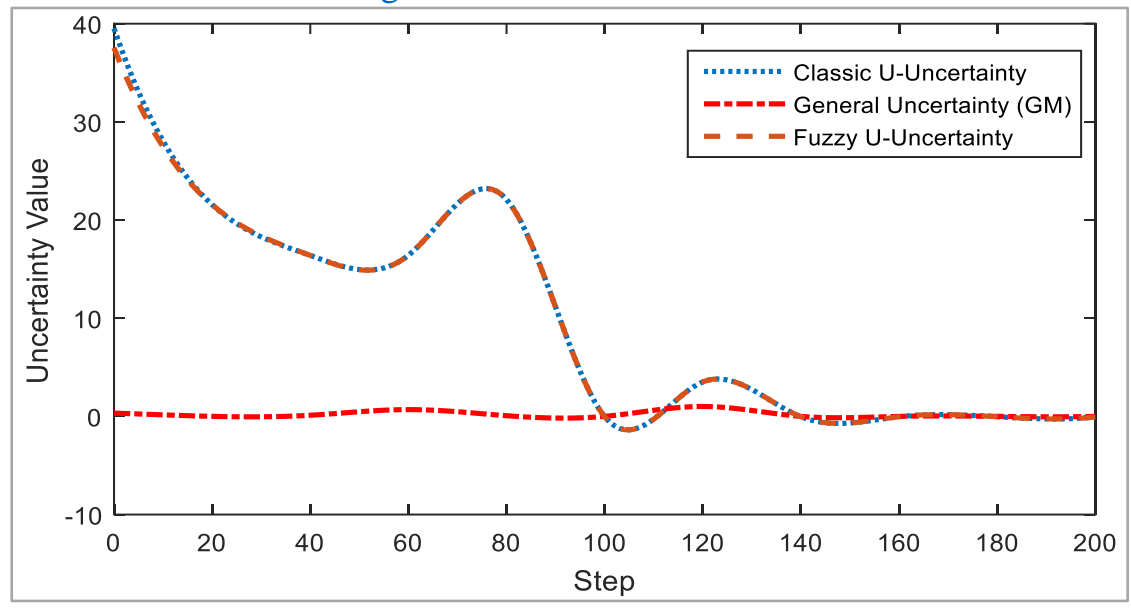

Fig. 11. The comparison of total uncertainty in FST and GFDST

Table 2. The comparison of total uncertainty in FST and GFSDT

\begin{tabular}{cccc} 
Number & Methods & Measure & Total Uncertainty \\
\hline 1 & Classic Sets Theory & Nonspecificity & 39.51 \\
2 & Fuzzy Sets Theory & Nonspecificity & 37.54 \\
$\mathbf{3}$ & Fuzzy Dempster-Shafer Theory & General Uncertainty & $\mathbf{1 . 0 1}$ \\
\hline
\end{tabular}

In Table 2, the total uncertainty in the suggested method is stated using the Generalized Fuzzy Dempster-Shafer Theory (GFDST). As shown in Table 2, the general uncertainty measure (GM) has been improved the total uncertainty relative to the non-specificity measure (U) in the air defense system. In Table 3, the features of the three measures are compared together.

Table 3. The comparison of features in three methods

\begin{tabular}{cccc} 
Method & Total Uncertainty & Decrease of Uncertainty & Computational Accuracy \\
\hline Classic Sets Theory & Poor & Good & Weak \\
Fuzzy Sets Theory & Poor & Good & Weak \\
$\begin{array}{c}\text { Fuzzy Dempster-Shafer } \\
\text { Theory }\end{array}$ & Very Good & Excellent & Very Good \\
\hline
\end{tabular}




\section{Conclusion}

In this paper, a new method of the target threat evaluation and calculation of uncertainty is presented using of Generalized Fuzzy Dempster-Shafer Theory (GFDST). GFDST responds to two main problems; first, the theory allows the calculation all three types of uncertainty in information and second, the theory allows to determine the ranking of the targets. GFDST integrates the meaning of the DST with FST to calculate all three types of uncertainty in a model. The newly suggested theory and the model based on it, are used to model the real-time scenario for threat evaluation in the air defense system. The results are verified by the total uncertainty earned from classic sets theory, fuzzy sets theory, and compared to GFDST. The results show that this theory and the model based on it are reasonable, effective, accurate, and reliable in the calculation of uncertainty. The future task can be improving the target parameters and their membership functions, optimizing uncertainty in the system, modifying the model, comparing uncertainty measures in DST to GFDST uncertainty measures, and constructing and implementing a battle management system for target threat assessment.

\section{Acknowledgment}

The authors thank to M. Goharimanesh for assistance with the experiments and valuable discussion.

\section{Declarations}

Author contribution. All authors contributed equally to the main contributor to this paper. All authors read and approved the final paper.

Funding statement. The authors received no specific funding for this work.

Conflict of interest. The authors declare no conflict of interest.

Additional information. No additional information is available for this paper.

\section{References}

[1] Aselsan, “C4I System Solutions," 2016, available at: https://www.aselsan.com.tr/C4I_System_ Solutions_2637.pdf.

[2] M. Azak and A. E. Bayrak, "A new approach for Threat Evaluation and Weapon Assignment problem, hybrid learning with multi-agent coordination," in 2008 23rd International Symposium on Computer and Information Sciences, 2008, pp. 1-6, doi: 10.1109/ISCIS.2008.4717866.

[3] Y. Zhang, S. Huang, S. Guo, and J. Zhu, "Multi-sensor Data Fusion for Cyber Security Situation Awareness," Procedia Environ. Sci., vol. 10, pp. 1029-1034, 2011, doi: 10.1016/j.proenv.2011.09.165.

[4] B. Li, "Navigation risk assessment scheme based on fuzzy Dempster-Shafer evidence theory," Int. J. Adv. Robot. Syst., vol. 15, no. 5, p. 172988141879957, Sep. 2018, doi: 10.1177/1729881418799572.

[5] N. Sadeghi, A. R. Fayek, and W. Pedrycz, "Fuzzy Monte Carlo Simulation and Risk Assessment in Construction," Comput. Civ. Infrastruct. Eng., vol. 25, no. 4, pp. 238-252, May 2010, doi: 10.1111/j.1467-8667.2009.00632.x.

[6] M. Raikhan, K. Bolat, Z. Meiram, and O. Altynay, "Assessing information security risk with the fuzzy set theory," J. Theor. Appl. Inf. Technol., 2018, available at: Google Scholar.

[7] Y. Pan, L. Zhang, Z. Li, and L. Ding, "Improved Fuzzy Bayesian Network-Based Risk Analysis With Interval-Valued Fuzzy Sets and D-S Evidence Theory," IEEE Trans. Fuzzy Syst., pp. 1-1, 2019, doi: 10.1109/TFUZZ.2019.2929024.

[8] H. M. Feng, X. F. Li, and J. F. Chen, "A comparative study of four Fuzzy Integrals for classifier fusion," in 2010 International Conference on Macbine Learning and Cybernetics, ICMLC 2010, 2010, doi: 10.1109/ICMLC.2010.5581040.

[9] K. Zhang, W. Kong, P. Liu, J. Shi, Y. Lei, and J. Zou, "Assessment and sequencing of air target threat based on intuitionistic fuzzy entropy and dynamic VIKOR," J. Syst. Eng. Electron., vol. 29, no. 2, pp. 305-310, Apr. 2018, doi: 10.21629/JSEE.2018.02.11. 
[10] M. R. Delavar and M. Sadrykia, "Assessment of Enhanced Dempster-Shafer Theory for Uncertainty Modeling in a GIS-Based Seismic Vulnerability Assessment Model, Case StudyTabriz City," ISPRS Int. J. Geo-Information, vol. 9, no. 4, p. 195, Mar. 2020, doi: 10.3390/ijgi9040195.

[11] M. Tavana, D. A. Trevisani, and D. T. Kennedy, "A Fuzzy cyber-risk analysis model for assessing attacks on the availability and integrity of the military command and control systems," 2015, doi: 10.4018/978-1-4666-7456-1.ch053.

[12] M. Yazdi and S. Kabir, "Fuzzy evidence theory and Bayesian networks for process systems risk analysis," Hum. Ecol. Risk Assess. An Int. J., vol. 26, no. 1, pp. 57-86, Jan. 2020, doi: 10.1080/10807039.2018.1493679.

[13] T. Lampinen, J. Ropponen, and T. Laitinen, "Joint threat assessment with asset profiling and entity bayes net," in 2009 12th International Conference on Information Fusion, FUSION 2009, 2009, available at: https://ieeexplore.ieee.org/abstract/document/5203731.

[14] Y. Liang, "A Fuzzy Knowledge Based System in Situation and Threat Assessment.," J. Syst. Sci. Inf., vol. 4, no. 4, 2006, available at : Google Scholar.

[15] M. J. Liebhaber and B. Feher, “Air Threat Assessment: Research, Model, and Display Guidelines,” Jan. 2002, doi: 10.21236/ADA458047.

[16] H. Liu, Z. Ma, X. Deng, and W. Jiang, "A new method to air target threat evaluation based on Dempster-Shafer evidence theory," in 2018 Chinese Control And Decision Conference (CCDC), 2018, pp. 2504-2508, doi: 10.1109/CCDC.2018.8407546.

[17] Z. Shu, "Target Ship Identification Algorithm Based on Comprehensive Correlation Discriminant and Information Entropy," J. Comput. Commun., vol. 08, no. 03, pp. 61-71, 2020, doi: 10.4236/jcc.2020.83007.

[18] J. Roux and J. Van Vuuren, “Threat evaluation and weapon assignment decision support: A review of the state of the art," ORiON, vol. 23, no. 2, Dec. 2007, doi: 10.5784/23-2-54.

[19] S. Kumar and A. M. Dixit, "Threat evaluation modelling for dynamic targets using fuzzy logic approach," in International Conference on Computer Science and Engineering, 2012, pp. 143-149, available at : Google Scholar.

[20] A. Burkov, S. Paquet, G. Michaud, and P. Valin, "An empirical study of uncertainty measures in the fuzzy evidence theory," in 14th International Conference on Information Fusion, 2011, pp. 1-8, available at : https://ieeexplore.ieee.org/abstract/document/5977677.

[21] A. Sarabi-Jamab and B. N. Araabi, "An information-based approach to handle various types of uncertainty in fuzzy bodies of evidence," PLoS One, 2020, doi: 10.1371/journal.pone.0227495.

[22] Hatefi, Basiri, and Tamošaitienė, "An Evidential Model for Environmental Risk Assessment in Projects Using Dempster-Shafer Theory of Evidence," Sustainability, vol. 11, no. 22, p. 6329, Nov. 2019, doi: 10.3390/su11226329.

[23] J. Huang, B. C. Li, and Y. J. Zhao, "Target Threat Assessment Based on Intuitionistic Fuzzy Sets Choquet Integral," Appl. Mech. Mater., vol. 433-435, pp. 736-743, Oct. 2013, doi: 10.4028/www.scientific.net/AMM.433-435.736.

[24] T. Ali and P. Dutta, "Methods to obtain basic Probability Assignment in Evidence Theory," Int. J. Comput. Appl., vol. 38, no. 4, pp. 46-51, Jan. 2012, doi: 10.5120/4600-6802.

[25] H. Zhang, J. Xie, Y. Song, J. Ge, and Z. Zhang, "A novel ranking method for intuitionistic fuzzy set based on information fusion and application to threat assessment," Iran. J. Fuzzy Syst., vol. 17, no. 1, pp. 91-104, 2020, available at: Google Scholar.

[26] M. Gul and A. F. Guneri, "A fuzzy multi criteria risk assessment based on decision matrix technique: A case study for aluminum industry," J. Loss Prev. Process Ind., vol. 40, pp. 89-100, Mar. 2016, doi: 10.1016/j.jlp.2015.11.023.

[27] P. Dutta, "Fuzzy-DSS Human Health Risk Assessment Under Uncertain Environment," 2018, pp. 316-347, doi: 10.4018/978-1-5225-5396-0.ch015. 
[28] A. Maseleno, M. M. Hasan, N. Tuah, and C. R. Tabbu, "Fuzzy Logic and Mathematical Theory of Evidence to Detect the Risk of Disease Spreading of Highly Pathogenic Avian Influenza H5N1," in Procedia Computer Science, 2015, doi: 10.1016/j.procs.2015.07.349.

[29] A. Lepskiy and A. Suevalov, "Application of the Belief Function Theory to the Development of Trading Strategies,” Procedia Comput. Sci., vol. 162, pp. 235-242, 2019, doi: 10.1016/j.procs.2019.11.280.

[30] W. Mei, "Air defense threat evaluation using fuzzy Bayesian classifier," in IJCCI 2013 - Proceedings of the 5th International Joint Conference on Computational Intelligence, 2013, doi: $10.5220 / 0004512602270232$.

[31] B. M. Ayyub and G. J. Klir, Uncertainty Modeling and Analysis in Engineering and the Sciences, 2006, doi: 10.1201/9781420011456.

[32] Y. Yang, D. Han, and J. Dezert, "A new non-specificity measure in evidence theory based on belief intervals," Chinese J. Aeronaut., vol. 29, no. 3, pp. 704-713, Jun. 2016, doi: 10.1016/j.cja.2016.03.004.

[33] E. Azimirad and J. Haddadnia, "A New Data Fusion Instrument for Threat Evaluation Using of Fuzzy Sets Theory,” Int. J. Comput. Sci. Inf. Secur., vol. 13, no. 4, p. 19, 2015, available at : Google Scholar.

[34] P. Dutta and T. Ali, "Fuzzy focal elements in dempster-shafer theory of evidence: case study in risk analysis," Int. J. Comput. Appl., vol. 34, no. 1, 2011, available at : Google Scholar.

[35] E. Azimirad and J. Haddadnia, "A new model for threat assessment in data fusion based on fuzzy evidence theory," Int. J. Adv. Intell. Informatics, vol. 2, no. 2, p. 54, Jul. 2016, doi: 10.26555/ijain.v2i2.56. 